\title{
Percutaneous Left Atrial Appendage Occlusion for the Prevention of Stroke in Patients with Atrial Fibrillation: Review and Critical Appraisal
}

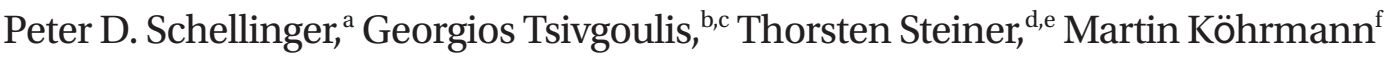 \\ aDepartment of Neurology and Neurogeriatry, Johannes Wesling Medical Center Minden, University Clinic RUB, Minden, Germany \\ ${ }^{b}$ Second Department of Neurology, Attikon University Hospital, National and Kapodistrian University of Athens, Athens, Greece \\ 'Department of Neurology, University of Tennessee Health Sciences Center, Memphis, TN, USA \\ ${ }^{d}$ Department of Neurology, Frankfurt Hoechst Hospital, Frankfurt, Germany \\ eDepartment of Neurology, Heidelberg University Hospital, Heidelberg, Germany \\ fDepartment of Neurology, Essen University Hospital, Essen, Germany
}

The authors review the current status of percutaneous left atrial appendage (LAA) occlusion therapy in patients with atrial fibrillation with the goal to prevent ischemic stroke and systemic embolism and to reduce oral anticoagulation associated bleeding. While we cover the historical and also surgical background, and all tested devices, the main focus rests on the single currently U.S. Food and Drug Administration (FDA) approved LAA occluder, the WATCHMAN device, and its approval process. The authors also give a critical appraisal beyond the review of mere facts, trying to put the current data into perspective.

Keywords Atrial fibrillation; Stroke; Atrial appendage occlusion; Brain ischemia; Intracranial hemorrhage

\author{
Correspondence: Peter D. Schellinger \\ Department of Neurology and \\ Neurogeriatry, Johannes Wesling Medical \\ Center Minden, University Clinic RUB, \\ Hans-Nolte-Str. 1, D-32429 Minden, \\ Germany \\ Tel: +49-571-7903501 \\ Fax: +49-571-790293500 \\ E-mail: peterschellinger@ \\ muehlenkreiskliniken.de \\ Received: September 8, 2018 \\ Revised: September 11, 2018 \\ Accepted: September 12, 2018
}

\section{Introduction and background}

"Quis custodiet ipsos custodes?" (Who watches the Watchmen?, Juvenal, Roman Satirist, approximately 100 AD, Aquinum, Satire $\mathrm{Vl}$, Rhyme 347). Atrial fibrillation (AF) has a prevalence of $1 \%$ in the general population and therefore is the most frequent cardiac rhythm disorder. ${ }^{1}$ Due to an increasing prevalence with increasing age, the absolute number of affected patients is expected to double over the next 30 years. Atrial thrombus formation is caused by prothrombotic processes among these increased adhesivity of the endocardium, stasis, and angiotensinmediated processes. ${ }^{2}$ The left atrial appendage (LAA) is an embryological remnant developing in the third week of gestation, its shape being variable, typically being a long tubular and hooked structure with different lobes and variable anatomy. ${ }^{3}$ It functions during conditions of volume overload as a reservoir and mediator of adaptive responses to decrease circulating blood volume mainly via atrial natriuretic peptide secretion. ${ }^{4}$ In AF the LAA can oscillate at rates faster than $300 /$ minute leading to a substantially reduced outflow and ultimately stasis. A systematic review by Blackshear and Odel| $\left.\right|^{5}$ analyzing 23 studies identified the LAA as source of embolic thrombi in more than $91 \%$ of patients with nonvalvular AF as opposed to $57 \%$ only in patients with rheumatic $A F$, establishing the rationale for $L A A$ obliteration as a preventive strategy for stroke in nonvalvular AF. However, a more recent analysis showed that while in valvular AF more than half the thrombi were located in the left atrial cavity also in patients with nonvalvular AF and with a history of stroke, in cases of the lack of anticoagulation or ventricular dysfunction the chances of left atrial cavity as opposed to LAA thrombus were up 
to $45 \% .{ }^{6}$ Further trials established a correlation of varying shapes such as 'chickenwing,' 'windsock', 'cactus', and 'cauliflower' with embolic risk, the chickenwing configuration being associated with the lowest risk. ${ }^{3,7}$ Protagonists of left atrial appendage occlusion (LAAO) have characterized the LAA as the 'most lethal human attachment, ${ }_{1}^{8}$ a term and assessment that has not been shared by everybody.

\section{Therapeutic approaches}

In $A F$, primary and secondary prevention of mainly cerebral and peripheral embolism is routinely achieved with vitamin $\mathrm{K}$ antagonists (VKAs) such as warfarin or coumadin derivatives, or, more recently with a variety of non-VKA oral anticoagulant medications (NOACs) such as dabigatran, rivaroxaban, apixaban, and edoxaban. ${ }^{9-11}$ Unanimously, NOACs have shown non-inferiority, in single instances even superiority for stroke prevention while having significantly less bleeding complications (intra- and extracranially). Despite the introduction of these new drugs for stroke prevention, many patients and physicians continue to seek alternatives for a variety of reasons, including contraindications, medication side effects, adherence and quality-of-life concerns. ${ }^{12}$ Based on the aforementioned assumption that over $90 \%$ of left atrial thrombi originate from the LAA, surgical LAA removal decades ago was a routinely used procedure in open heart surgery for other indications (comparable to preventive appendix removal in otherwise indicated abdominal surgery) and first performed in $1948 .{ }^{13}$ Still, LAA removal was and is performed more recently, e.g., during mitral valve surgery or maze procedures (series of incisions arranged in a maze-like pattern in the atria). ${ }^{5}$ While the feasibility of surgical LAAO has been investigated, ${ }^{14,15}$ it has not been clarified whether the frequent incompleteness of LAAO during other cardiac surgery procedures does not ultimately lead to an even higher thrombogeneity and increasing rate of embolic complications. ${ }^{16}$ Furthermore, a primary surgical occlusion, even if performed via thoracoscopy, appears not to be justifiable at the current stage of research. ${ }^{17}$

At least eight transcutaneous LAAO devices have been developed over the last 20 years. ${ }^{18}$ Some of these are not approved or Conformité Européene (CE)-marked, while others are currently under evaluation in clinical trials, or have very few data at present. All of them (except the LARIAT device named for a rope used as a lasso or for tethering) share some common features for the procedure. At first extensive transesophageal echocardiography workup is performed to exclude intraluminal
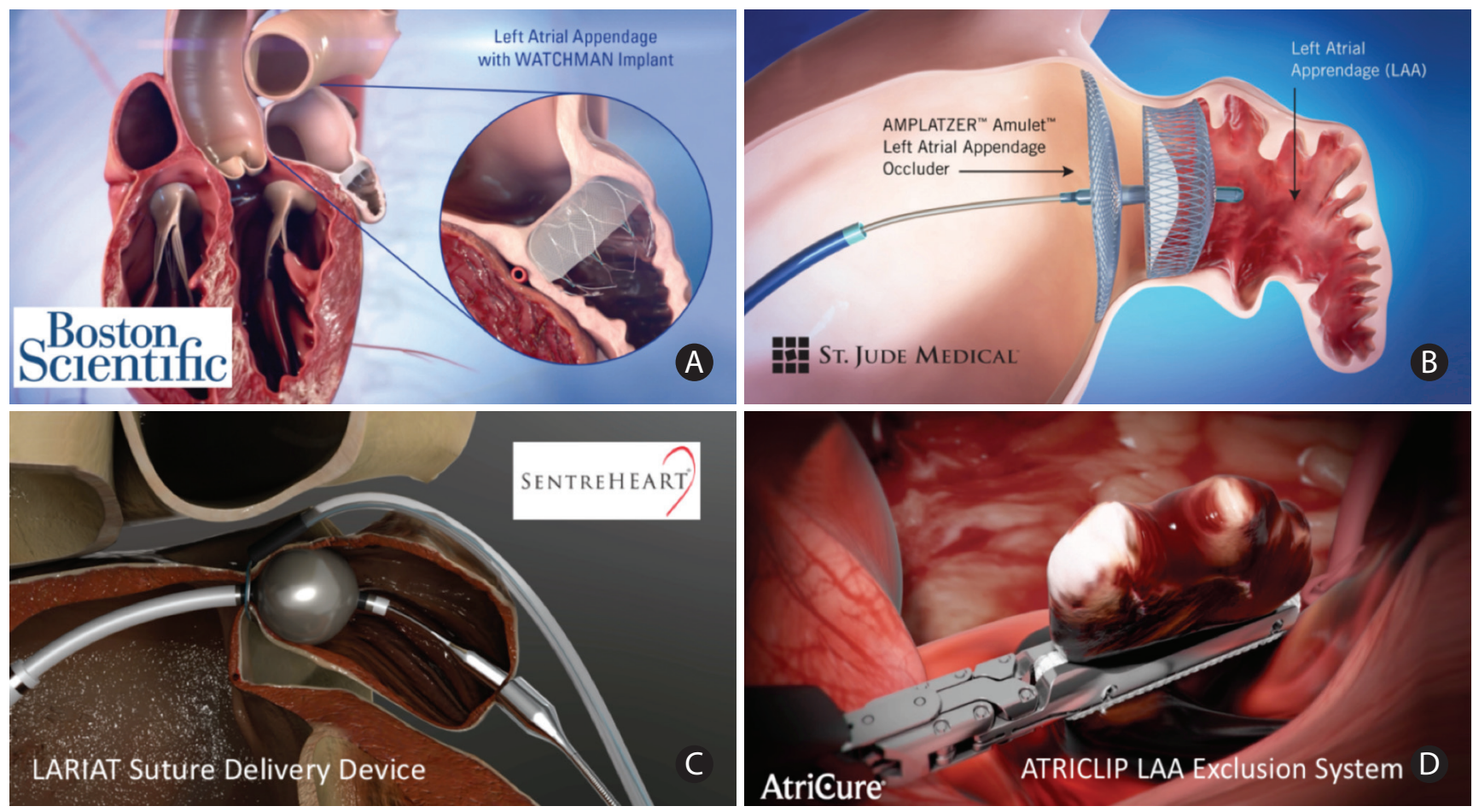

Figure 1. Left atrial appendage (LAA) closure devices and schematics of their deployment. Different types of LAA closure devices are seen. Endocardial devices include (A) WATCHMAN ${ }^{\mathrm{TM}}$ (image provided courtesy of Boston Scientific, c2018 Boston Scientific Corporation or its affiliates, http://www.bostonscientific.com) and (B) AMPLATZER ${ }^{\text {TM }}$ AMULET ${ }^{\text {TM }}$ (reproduced with permission of St. Jude Medical, c2018, https://www.sjmglobal.com). (C) The hybrid (endocardial and epicardial) LARIAT ${ }^{T M}$ suture delivery system for LAA exclusion (reproduced with permission of SENTREHEART, c2018, http://www.sentreheart.com) and (D) AtriClip ${ }^{T M}$ for surgical clipping (reproduced with permission of AtriCure, c2018, https://www.atricure.com). Devices are trademarks of their respective companies, all rights reserved. Adapted from Topcuoglu et al. ${ }^{22}$ 
thrombi or other pathologies and characterize the anatomy of the LAA. The procedure itself involves anesthesia, catheterization of the femoral vein and access to the LAA via the right atrium and a transseptal access, i.e., puncture and penetration of the septum with an e.g., 14 French $(4.7 \mathrm{~mm})$ catheter system. Only newer systems such as the LAmbre system use smaller 8 to 10 French ( 2.7 to $3.3 \mathrm{~mm}$ ) introducer sheaths. For more technical details the reader is referred to the following references. ${ }^{19-21}$

Figure 1 taken from Topcuoglu et al. ${ }^{22}$ in 2018 illustrates some of the devices and their anatomical relation to the LAA.

\section{Devices outside randomized controlled trials}

None of the devices except the WATCHMAN LAA occluder have been tested in a randomized fashion. Therefore the WATCHMAN data will be dealt with in more detail.

\section{PLAATO (Percutaneous Left Atrial Appendage} Transcatheter Occlusion) device

This first generation device was a self expanding nitinol cage with anchors to prevent embolization. ${ }^{23}$ Testing in a non-randomized series of 111 patients with successful occlusion in 108 patients with four severe complications achieved a reduction of stroke rates compared to Congestive heart failure, Hypertension, Age, Diabetes, Stroke history $\left(\mathrm{CHADS}_{2}\right)$-score predicted stroke rates. ${ }^{24}$ This was followed by a combined report of 180 patients, the later trial being halted. ${ }^{25}$ After increasing reports of complications the device production was halted and never reached a commercial level for 'marketing reasons,' a courageous decision considering that it was the company's only product at that time in this market.

\section{Amplatzer cardiac plug and Amulet device}

For the amplatzer cardiac plug (ACP) device only case series ${ }^{26}$ and a 'post marketing observational study' were performed. ${ }^{27} \mathrm{~A}$ truly randomized trial was planned (as for PLAATO) according to ClinicalTrials.gov but never recruited any patients. In the latter series by Tzikas et al., ${ }^{27}$ 1,047 patients were collected in 22 centers with self reported (non-adjudicated) procedure success in 97.3\% and 52 (4.97\%) major adverse events. Again the expected stroke rate according to $\mathrm{CHADS}_{2}$-scores was approximately 5\% and then reduced to 2.1\%. The Amplatzer Amulet device has succeeded the ACP in most instances ${ }^{28}$ and is tested in two randomized trials (Amulet Investigational Device Exemption [IDE] against WATCHMAN device; Prevention of Stroke by Left Atrial Appendage Closure in Atrial Fibrillation Pati- ents After Intracerebral Hemorrhage [STROKECLOSE] trial against best medical treatment after intracerebral hemorrhage). The most recent data come from a large prospective registry published by Landmesser et al., ${ }^{29}$ where 1,088 patients have been included. A new aspect in this registry was that average Congestive heart failure, Hypertension, Age, Diabetes, Stroke history, Vascular disease, Age, Sex category ( $\mathrm{CHA}_{2} \mathrm{DS}_{2}$-VASc) scores were relatively high at 4.2 points and that $83 \%$ of patients had contraindications to oral anticoagulant (OAC) plus $72 \%$ had a history of major bleeding. There was no residual flow in $98.4 \%$ of patients and the observed ischaemic stroke rate was $2.9 \%$ per year. A device-related thrombus was noted in $1.7 \%$ of patients. In the first year, major bleeding occurred at an annualised rate of $10.3 \%$ and all-cause mortality was $8.4 \%$. As there was no active comparator we are not able to say whether that is good or not in terms of efficacy or safety. In a propensity matched comparison of patients with AF and a history of intracranial hemorrhage (ICH), treated with Amplatzer LAAOs compared to similar patients treated with standard therapy, the composite outcome of mortality, ischemic stroke, and major hemorrhage was reduced for all patients (151 patients per group) and for those on OAC (103 patients per group). Percutaneous LAA closure was associated with a significant $84 \%$ reduction in the risk of the composite outcome compared to standard therapy and $74 \%$ in those on OAC therapy within 180 days after the index $\mathrm{ICH}^{30}$

\section{Next generation devices}

Newer devices include the CE-marked WaveCrest Left Atrial Appendage Occlusion System (Coherex Medical, Salt Lake City, UT, USA), the ultrasept LAA closure device, the LAmbre LAA Closure system (Lifetech Scientific, Shenzhen, China), and the Occlutech LAA occluder (the latter two also CE-marked; Occlutech, Helsingborg, Sweden). ${ }^{18,31,32}$ For the Occlutech and LAmbre devices small series with 30 and 60 patients respectively have been published, showing results generally in line with the other publications. ${ }^{33,34}$ The TigerPaw II System (Laax, Livermore, CA, USA) has had a major U.S. Food and Drug Administration (FDA) recall in 2015 and to our knowledge is not further developed. The AtriClip device (AtriCure, Mason, OH, USA) is used during open heart surgery and therefore not within the scope of this review.

\section{Lariat suture device}

Another transcatheter but combined endo-/epicardial LAA closure device is the Lariat Suture Delivery System (SentreHEART, Redwood City, CA, USA), which is used to ligate the LAA placing a knot around the ostium of the LAA and then removes the LAA. ${ }^{18}$ After percutaneous epicardial access and alignment with 
a magnet tipped wire delivered endocardially with a transseptal approach, an appendage grabber with embedded electrodes within the jaws permits electrical navigation and identification of the LAA, and finally after placing the loop and suture allows confirmation of firm closure by the elimination of LAA electrical activity. ${ }^{21}$ The current literature is sparse; however, recent comparative data to the WATCHMAN device were promising. A multicenter registry published by Lakkireddy et al. ${ }^{35}$ with 712 consecutive patients undergoing LAA ligation with LARIAT at 18 United States hospitals showed successful deployment in 682 patients (95.5\%) and complete closure in 669 patients (98\%). The two main complications were perforations in 3.5\% necessitating surgery in $1.44 \%$, and pericarditis in $4.78 \%{ }^{35} \mathrm{~A}$ recent publication assessed the safety of WATCHMAN $(n=5,849)$ and LARIAT $(n=4,889)$ devices by analysis of surveillance data from the FDA Manufacturer and User Facility Device Experience (MAUDE) database. ${ }^{36}$ Interestingly, the cumulative incidence of safety events (composite outcome of stroke/transient ischemic attack [TIA], pericardiocentesis, cardiac surgery, and death) occurred more frequently with WATCHMAN (cumulative incidence, $1.93 \%$ vs. $1.15 \% ; P=0.001) .^{36}$ Another device, the Sierra Ligation System (Aegis Medical Innovations, Vancouver, BC, Canada) is completely extravascular/epicardial, and therefore not covered in this report.

\section{The WATCHMAN trials}

The WATCHMAN LAAO device was tested in two randomized trials, the "WATCHMAN Left Atrial Appendage System for Embolic Protection in Patients With Atrial Fibrillation" (PROTECT-AF) trial and the "Evaluation of the WATCHMAN LAA Closure Device in Patients With Atrial Fibrillation Versus Long Term Warfarin Therapy" (PREVAIL) study, ${ }_{1}^{37,38}$ a prospective registry (continued access protocol [CAP]), ${ }_{1}^{39}$ a German/Swiss registry (efficacy and safety of left atrial appendage closure with WATCHMAN in patients with or without contraindication to oral anticoagulation [EWOLUTION] $)^{12,40}$ and several follow-up analyses of PROTECT-AF ${ }^{39,41}$ (Table 1). The only randomized evidence (with a few caveats) is derived from the original PROTECT-AF trial and its longterm follow-up analyses.

\section{PROTECT-AF}

PROTECT-AF assessed the efficacy and safety of percutaneous closure of the LAA for prevention of stroke compared with warfarin treatment in adult patients with $\mathrm{AF}$ in a multicentre, randomised non-inferiority trial. Inclusion criteria were a $\mathrm{CHADS}_{2}$ score of at least 1 point, exclusion criteria were contraindications to warfarin, necessity of chronic warfarin use, LAA thrombus, patent foramen ovale with atrial septal aneurysm (ASA), and symptomatic carotid artery disease. Seven hundred and seven patients were randomized 2:1 (intervention, $n=463$; cont-

Table 1. Summary of clinical studies for the WATCHMAN left atrial appendage occluder

\begin{tabular}{lcccc}
\hline & PROTECT-AF & CAP registry & PREVAIL & EWOLUTION \\
\hline Enrollment & $2005-2008$ & $2008-2010$ & $2010-2012$ & $2013-2015$ \\
Study design & Randomized & Registry & Randomized & Registry \\
Randomization & $2: 1$ & NA & Warfarin & NA \\
Control & Warfarin & NA & 407 & 1,025 \\
Number of patients & 707 & 460 & $74 \pm 7.4$ & $73.4 \pm 9$ \\
Age & $72 \pm 8.9$ & $74 \pm 8.3$ & $2.6 \pm 1.0$ & $2.8 \pm 1.3$ \\
CHADS $_{2}$ & $2.2 \pm 1.2$ & $2.5 \pm 1.2$ & $3.8 \pm 1.2$ & $4.5 \pm 1.6$ \\
CHA $_{2}$ DS 2 -VASc & $3.3 \pm 1.4$ & $3.7 \pm 1.4$ & 28 & 30.5 \\
Stroke/TIA (\%) & 18 & 30 & 54 & 50.8 \\
Age $\geq 75$ yr (\%) & 43 & 52 & 400 & $1 \mathrm{yr}$ \\
Follow-up (patient-yr) & 2,621 & 1,328 & 0.98 & NA \\
Mean duration (yr) & $3.8^{*}$ & 2.4 & 95 & 98.5 \\
Implant success (\%) & 91 & 94 & 4.2 & 2.7 \\
7-Day procedure related SAEs (\%) & 8.7 & 4.1 & & \\
\hline
\end{tabular}

PROTECT-AF, WATCHMAN Left Atrial Appendage System for Embolic Protection in Patients With Atrial Fibrillation; CAP, continued access protocol; PREVAIL, Evaluation of the WATCHMAN LAA Closure Device in Patients With Atrial Fibrillation Versus Long Term Warfarin Therapy; EWOLUTION, efficacy and safety of left atrial appendage closure with WATCHMAN in patients with or without contraindication to oral anticoagulation; NA, not available; CHADS $2_{1}$ Congestive heart failure, Hypertension, Age, Diabetes, Stroke (doubled); $\mathrm{CHA}_{2} \mathrm{DS}_{2}$-VASc, Congestive heart failure, Hypertension, Age, Diabetes, Stroke history, Vascular disease, Age, Sex category; TIA, transient ischemic attack; SAE, serious adverse event.

*Longterm follow-up, initial publication 2009. 
rol, $n=244$ ) for LAAO versus warfarin with a target international normalized ratio (INR) of 2 to 3 . Patients with LAAO were treated with warfarin for 45 days, followed by clopidogrel and aspirin for 6 months and if possible ASA alone after 7.5 months. Efficacy was assessed by a primary composite endpoint of stroke, cardiovascular death, and systemic embolism. For this endpoint a $>99.9 \%$ probability of non-inferiority was achieved albeit not for superiority. It needs to be mentioned that primary safety events were more frequent in the intervention group than in the control group (7.4 per 100 patient-years [95\% confidence interval $(\mathrm{Cl}), 5.5$ to 9.7 ] vs. 4.4 per 100 patient-years [95\% Cl, 2.5 to 6.7 ]; rate ratio [RR], $1.69,1.01$ to 3.19 ). Also, the prevention of ischemic strokes was not non-inferior (probablity of $71.8 \%$ ), periprocedural strokes occured in $1.1 \%$, ICH rates in the warfarin arm were surprisingly high with $2.5 \%$, and pericardiac tamponade requiring surgical or percutaneous treatment occurred in 4.8\%.

A 4-year follow-up through October 2012 was published in 2014 by Reddy et al. ${ }^{41}$ At a mean follow-up of $3.8 \pm 1.7$ years (2,621 patient-years), there were 39 events among 463 patients $(8.4 \%)$ in the device group for a primary event rate of 2.3 events per 100 patient-years, compared with 34 events among 244 patients (13.9\%) for a primary event rate of 3.8 events per 100 patient-years with warfarin ( $\mathrm{RR}, 0.60 ; 95 \% \mathrm{Cl}, 0.41$ to 1.05$)$, meeting prespecified criteria for both noninferiority (posterior probability, >99.9\%) and superiority (posterior probability, 96.0\%). ${ }^{41}$ This was accompanied by a significant reduction of both cardiovascular mortality (3.7\% vs. 9.0\%; hazard ratio, $0.40 ; 95 \% \mathrm{Cl}$, 0.21 to $0.75 ; P=0.005$ ), and all-cause mortality. The authors concluded that percutaneous LAA closure with the WATCHMAN device met criteria for both noninferiority and superiority, compared with warfarin, for preventing the combined outcome of stroke, systemic embolism, and cardiovascular death, as well as superiority for cardiovascular and all-cause mortality. ${ }^{41}$

Several aspects of PROTECT-AF were critically discussed. ${ }^{42-45}$ The results could be put into perspective in a different way. For 100 patient-years, the ischemic stroke rate with WATCHMAN was non-significantly increased (2.2\% vs. $1.6 \%)$ and severe complications were significantly more frequent (7.4 vs. 4.4 per 100 patient-years), only intracranial bleedings being less frequent in the WATCHMAN arm (0.1 vs. 1.6 per 100 patient-years). However, the rate of ICH in the warfarin arm was substantially higher $(2.5 \%)$ than in the warfarin arms of the recent NOAC trials (0.4\% to $0.75 \%$ ) with comparable $\mathrm{CHADS}_{2}$-scores. ${ }^{9-11}$ Also, bleeding rates are not different anymore, when procedure associated bleedings are incorporated (and not counted starting after day 7). Less than $20 \%$ of patients were included in a secondary prophylactic indication, i.e., with a his- tory of TIA or stroke (68\% of patients were low risk patients with a $\mathrm{CHADS}_{2}$ score of 1 or 2), and of the 408 'successful' treatments warfarin could be terminated in only 355 patients after day 45 because of peridevice leakage (76.6\%). The latter is equal to an incomplete occlusion of the LAA, which again potentially does not reduce or may even increase thrombogeneity of the LAA. The number needed to harm was 20 for pericardial effusion, 50 for emergency open heart surgery, and 100 for periprocedural stroke. Also after 3 years, non-inferiority was shown for the primary endpoint only, but neither ischemic stroke alone nor safety. The FDA decision regarding approval of the WATCHMAN device at that point (see below) necessitated further research.

\section{CAP (and PROTECT-AF)}

In addition to the PROTECT-AF data more information regarding safety events and the potential for improving procedural safety were required, which is the basis for the ongoing CAP registry. ${ }^{39}$ For this analysis, patients from the PROTECT-AF trial who underwent attempted LAA closure ( $\mathrm{n}=542$ patients) and from the CAP ( $n=460$ patients) were included (Table 1). The authors observed a significant decline in the rate of procedure- or device-related safety events within 7 days of the procedure across the two studies, with $7.7 \%$ and $3.7 \%$ of patients, respectively, experiencing events $(P=0.007)$, and between the first and second halves of PROTECT-AF and CAP registry, with $10.0 \%$, 5.5\%, and $3.7 \%$ of patients, respectively, experiencing events $(P=0.006)$. Also, the rate of serious pericardial effusion within 7 days of implantation, which had made up $>50 \%$ of the safety events in PROTECT-AF, was lower in the CAP registry $(5.0 \%$ vs. $2.2 \%$, respectively; $P=0.019) .{ }^{39}$ These results were put into another perspective by arbitrarily defining a net clinical benefit formula and include patients from the PROTECT-AF trial and another 566 patients in the CAP registry in a post hoc analysis of outcomes. ${ }^{46}$ The outcomes ischemic stroke, intracranial haemorrhage, major bleeding, pericardial effusion, and death were weighted to reflect the relative impact in terms of death and disability. Thereby the authors calculated a net clinical benefit as the sum of annualized rates of 1.73\% per year for the trial ( $95 \% \mathrm{Cl},-0.54 \%$ to $4.39 \%$ per year) and $4.97 \%$ per year for the CAP ( $95 \% \mathrm{Cl}, 3.07 \%$ to $7.15 \%$ per year). Interestingly, among patients with a history of ischemic stroke, the net clinical benefit was larger in the registry (8.68\% per year; 95\% $\mathrm{Cl}, 2.82 \%$ to $14.92 \%$ per year) than the trial (4.30\% per year; $95 \% \mathrm{Cl}_{1}-2.07 \%$ to $11.25 \%$ per year). Also the net clinical benefit was larger in patients with higher $\mathrm{CHADS}_{2}$-scores. ${ }^{46}$ 


\section{ASAP registry}

The aspirin and plavix (ASAP) registry prospectively collected patients with contraindications for, or inability to tolerate warfarin therapy ( $n=150$ patients). Based on the "Atrial Fibrillation Clopidogrel Trial with Irbesartan for Prevention of Vascular Events (ACTIVE) A" trial ${ }^{47}$ a combination of clopidogrel and aspirin may have minimal preventive effect $(0.8 \%$ absolute) over aspirin alone for AF patients coming with a low level recommendation in European Society of Cardiology (ESC) guidelines due to similar bleeding risk as VKA but a much lower efficacy in preventing ischemic stroke or systemic embolism. ASAP was non-randomized and performed in four European centers. ${ }^{48}$ History of hemorrhagic/bleeding tendencies (93\%) was the most common reason for warfarin ineligibility. Mean duration of follow-up was $14.4 \pm 8.6$ months. The average $\mathrm{CHADS}_{2}$-score was 2.8, and clopidogrel was taken for 6 months while aspirin was taken for an indefinite amount of time after the LAAO procedure. The device was implanted succcessfully in 94.7\% (142/150 patients), and the observed stroke rate was $1.7 \%$. Serious procedure- or device-related safety events occurred in $8.7 \%$ of patients $\left(13 / 150\right.$ patients). ${ }^{48}$

\section{PREVAIL}

The PREVAIL study was performed to confirm the results of the PROTECT-AF study also with regard to concerns raised with that trial. ${ }^{38}$ The design was similar (Table 1) to PROTECT-AF, and a total of 407 patients (intervention group, $n=269$; control group, $n=138$ ) were recruited, also in a 2:1 randomization fashion versus warfarin. Mildly higher implantation success rates were found (95.1\% vs. $90.9 \%$ ) with a lower complication rate. Two efficacy and one safety endpoints were assessed. The efficacy coprimary endpoints were (1) composite of stroke, systemic embolism, and cardiovascular/unexplained death, and (2) stroke or systemic embolism $>7$ days postrandomization. For the first coprimary endpoint, non-inferiority could not be demonstrated, for the second endpoint only non-inferiority but no superiority was shown. Procedure- or device-related complications (safety endpoint) within 7 days occurred in $4.5 \%$ in PREVAIL compared to $8.7 \%$ in PROTECT-AF. Also pericardial effusions requiring surgical repair were significantly less frequent (0.4\% vs. $1.6 \%$ in PROTECT-AF). ${ }^{38}$

\section{EWOLUTION}

The EWOLUTION registry was another prospective registry to provide data in a routine clinical practice setting. ${ }^{12,40} \mathrm{~A}$ total of 1,025 patients scheduled for a WATCHMAN implant were prospectively enrolled; the indication for LAAO was based on cur- rent ESC guidelines. The population differed from the PROTECT-AF trial in terms of history of stroke/TIA and bleedings (Table 1). The $15.1 \%$ of patients had previous hemorrhagic stroke, and $320(31.3 \%)$ had a history of major bleeding; and $750(73 \%)$ were deemed unsuitable for oral anticoagulation therapy. At 1 year, mortality was $9.8 \%$, reflecting the advanced age and comorbidities of this population. Interestingly, compared to the other trials, complication rates during the first 7 days were low at $2.7 \%$ compared to $8.7 \%$ in PROTECT-AF. Ischemic stroke rate was 1.1\% (relative risk reduction of 84\% vs. estimated historical data esimated at 7.2\%). Major bleedings were reduced by $50 \%$ from $5 \%$ to $2.5 \%$ according to expected rates from Hypertension, Abnormal renal and liver function, Stroke, Bleeding, Labile INR, Elderly, Drugs or alcohol (HASBLED) scores. ${ }^{40}$ Again the authors conclude that with more experience, standardization, training, and establishment of the procedure, previously worrisome implantation success and even more worrisome complication rates improve significantly making the procedure a viable alternative to medical prevention of stroke, systemic embolism, and extra- as well as intracranial bleedings.

\section{Meta-analyses}

The most updated meta-analysis evaluating the safety and technical success of percutaneous LAAO using different devices included 49 studies involving 12,415 patients. The median age was 73.5 years (interquartile range [IQR], 72 to 75) and $43 \%$ were men. The median $\mathrm{CHADS}_{2}$ score was 2.9 (IOR, 2.6 to 3.3 ) and the median HASBLED score was 3.3 (IQR, 3 to 4). LAAO implantation was successful in $96.3 \%$ of patients (95\% Cl, 95.40 to $\left.97.08 ; I^{2}=76.1 \%\right)$. The pooled proportion of all-cause mortality was $0.28 \%\left(95 \% \mathrm{Cl}, 0.19\right.$ to $\left.0.38 ; \mathrm{I}^{2}=0 \%\right)$. The pooled proportion of all-cause stroke was $0.31 \%$ (95\% $\mathrm{Cl}, 0.22$ to $\left.0.42 ; I^{2}=9.4 \%\right)$, major bleeding requiring transfusion was $1.71 \%\left(95 \% \mathrm{Cl}, 1.13\right.$ to $\left.2.41 ; l^{2}=73.2 \%\right)$, and pericardial effusion was $3.25 \%\left(95 \% \mathrm{Cl}, 2.46\right.$ to $\left.4.14 ; \mathrm{I}^{2}=79 \%\right){ }^{49}$ Another systematic review and meta-analysis sought to compare LAAO to medical therapy using randomized controlled trial (RCT) data. This meta-analysis included five RCTs (three with surgical and two with percutaneous occlusion) and a total of 1,285 patients. LAAO failed to prevent stroke (RR, $0.78 ; 95 \% \mathrm{Cl}, 0.47$ to 1.29 ), but a significant reduction in mortality ( $\mathrm{RR}, 0.71 ; 95 \% \mathrm{Cl}, 0.51$ to 0.99$)$ was documented with LAAO. Notably, the quality of the included RCTs was assessed using the Grading of Recommendations, Assessment, Development and Evaluations (GRADE) system and was evaluated as poor. ${ }^{50}$ 


\section{The FDA process}

In 2015, Waksman and Pendyala ${ }^{45}$ published the overview of the FDA circulatory system devices panel meetings on WATCHMAN LAAO. The authors of this review strongly recommend the original reference for detailed reading on the approval process (and its inconsistencies) of the WATCHMAN device, the only FDA approved LAA occluder to date. This panel is an advisory board for the FDA and not deliberate to force decisions, albeit in general FDA is said to follow recommendations of such panels. The first review of the premarket approval application of the WATCHMAN based on the PROTECT-AF results took place in April 2009. Short term efficacy was accepted by the board but based on the short term periprocedural complication rates and safety issues with lacking longterm efficacy data the FDA issued a 'not approvable' letter. The next panel meeting took place in July 2010. 'Homework' for the sponsor besides the accumulation of registry and real life data (CAP registry) consisted of design and performance of a second randomized trial, i.e., the PREVAIL study. FDA recommended a Bayesian design which allows the borrowing of data from PROTECT-AF, a trial they deemed not sufficient for general approval of the WATCHMAN LAAO but still containing valuable information. This means that the closer the trial population of PREVAIL would fit that of PROTECT-AF, the more data could be borrowed and the more these data can be weighted.

Major concerns of the panel consisted of a low risk patient population (33\% had a $\mathrm{CHADS}_{2}$ score of 1), the mix of antiplatelet (combination) regimes, and their confounding effect, primary device associated safety events occurring on day one without this being formally tested as an outcome. All of these issues were addressed in the design of PREVAIL. PREVAIL was a small trial with 269 subjects randomized to the device grouporiginally a trial with more than 1,000 patients was expected to be done. PROTECT-AF data were incorporated with a weight of $50 \%$. PREVAIL failed to meet the non-inferiority criterion for the first primary endpoint and several panel members expressed their ongoing concerns, although overall the panel was mostly "in favor of using the totality of the data." The second endpoint in PREVAIL addressed longterm efficacy, i.e., 7 days (post-intervention) to 18 months rate of ischemic stroke and systemic embolism as a measure of mechanism of action. The positive test for non-inferiority "mitigated concerns about ischemic stroke rates." The sponsor proposed in the December 2013 panel meeting an indication for use as follows: "WATCHMAN LAA closure therapy is indicated to prevent thromboembolism from the LAA. It may be considered for use in patients with nonvalvular AF who are eligible for warfarin therapy to reduce the risk of stroke and systemic embolism based on $\mathrm{CHADS}_{2}$ or $\mathrm{CHA}_{2} \mathrm{DS}_{2}$-VASc scores." The panel members in contrast put an emphasis on warfarin therapy eligibility, $\mathrm{CHA}_{2} \mathrm{DS}_{2}-\mathrm{VASc}$ scores, and a personalized approach, including consideration of patient preference regarding long-term anticoagulation therapy. The panel also suggested adding the statement, "and who have reason not to remain on chronic warfarin therapy," to the end of the current indications for use. Furthermore, the panel believed that the following aspects should be included in the labelling (1) a contraindication for those ineligible for anticoagulation therapy, (2) a statement that there should be a reasonable expectation of patient survival, (3) consistency with clinical trial medication guidelines regarding use of antiplatelet therapy and anticoagulation therapy, and (4) a detailed characterization of attributes for success from experienced centers and implanters. ${ }^{45}$ At the end the panel voted 13:1 in favor of the device.

In Tables 5 and 6 of the Waksman and Pendyala ${ }^{45}$ new data since the December 2013 panel meeting are presented. Here, 13 new ischemic strokes in the device group (all $>1$ year post procedure) versus one new stroke in the control group shifted the efficacy outcomes towards inefficacy. Specifically, there were 24 primary end point events in the device group (13 ischemic strokes, two hemorrhagic strokes, one embolism, eight cardiovascular/unexplained deaths) and nine events in the control group (one ischemic stroke, two hemorrhagic strokes, and six cardiovascular or unexplained deaths). This caused a further divergence of PROTECT-AF and PREVAIL data, and even with incorporated (50\% discounted) PROTECT-AF data, non-inferiority could not be shown for either of the two endpoints. In other words, and the ischemic stroke rate control/device was $0.15(P=0.044)$ in favor of control, and the ischemic stroke and systemic embolism rate was $0.14(P=0.027)$, also in favor of control. After several meetings and discussions, and taking registry data into account, the panel finally voted as follows: 12 to 0 that the WATCHMAN LAA closure device is safe; 6 to 7 that it is not effective; and 6 to 5 , with 1 abstention, that its benefits outweigh its risks.

The FDA conditionally approved the WATCHMAN March 2015 with the following label. This device is indicated to reduce the risk of thromboembolism from the LAA in patients with nonvalvular AF who: (1) are at increased risk for stroke and systemic embolism based on $\mathrm{CHADS}_{2}$ or $\mathrm{CHA}_{2} \mathrm{DS}_{2}$-VASc scores and are recommended for anticoagulation therapy; (2) are deemed by their physicians to be suitable for warfarin; and (3) have an appropriate rationale to seek a non-pharmacologic alternative to warfarin, taking into account the safety and effectiveness of the device compared to warfarin. The final panel vote in May 2016 was 15 to 1 that the WATCHMAN LAAO de- 
vice is safe; 9 to 7 that it is effective; and 11 to 5 , that its benefits outweigh its risks.

\section{Critical appraisal}

First of all, the authors strongly believe that, e.g., therapeutic interventional, alternative to OAC, longterm options for patients with AF are dearly needed and development of these is absolutely justified. There is no need to throw the baby out with the bathwater. Unfortunately, the development process of LAA occluder devices is overshadowed by inconsistent data from randomized trials and registries for one device, i.e., the WATCHMAN, whereas actually several others are used without a label (or randomized data), e.g., ACP, Amulet, Lariat, or others. Favourable results based on lower than expected stroke rates according to $\mathrm{CHADS}_{2}$ or $\mathrm{CHA}_{2} \mathrm{DS}_{2}$-VAsc scores do not at all suffice as surrogate proof for efficacy and safety. Besides of all the issues discussed above and within the FDA panel report ${ }^{45}$ with the PROTECT-AF trial, the two major issues coming to our minds are: (1) the fact that even with borrowed data from PROTECT-AF, non-inferiority of the WATCHMAN could not be established for both coprimary endpoints in PREVAIL. To our understanding, not being able to prove the alternate hypothesis (of non-inferiority) in a one-sided test actually shows that the experimental arm (i.e., device) is actually inferior in terms of efficacy (the null hypothesis). Even if safety issues were substantially reduced over time with device improvement and training etc., the substantial doubt regarding efficacy raises the questing regarding whether the device should be used in the given label/indication; (2) a point also raised in the Waksman manuscript is the fact that to date no trial has compared a LAA occluder to oral anticoagulation with a NOAC. NOACs are associated with equal (better to say non-inferior) or higher preventive efficacy and a substantially lower bleeding rate in AF patients. This has been even shown compared to aspirin, in patients who are ineligible for VKAs. ${ }^{51}$ Even the FDA concluded that "WATCHMAN is better than no therapy or ineffective therapy," is "recommended as second line therapy," and may be "safe but not effective," ${ }^{45}$ therefore limited the actual labelling.

While registries may serve to illustrate real life experiences, they cannot substitute a randomized comparison and independently adjudicated safety and efficacy endpoints of a randomized trial. Introducing an arbitrary net benefit formula does not help in gaining trust. ${ }^{46,52}$ However, the sequential and substantial reduction in procedure related complications throughout CAP, PREVAIL, and EWOLUTION is plausible and encouraging. In our opinion, therefore the indication and contraindication as per approval should be carefully reviewed before patients un- dergo or are referred to a LAA closure procedure. While on many occasions LAAO is considered for patients not able to receive OAC (whether with VKA or NOACs), the current FDA approval reads differently, i.e., to the opposite. Only patients eligible for OAC should receive LAA closure. For all these reasons device-based therapies have been developed for stroke prevention in bleeding-prone, high-risk, patients with non-valvular AF and for those patients who cannot take or have substantial reason not to take oral anticoagulation therapies as well as patients having issues with adherence.

On a second thought, NOACs have not been compared to LAA occluders yet (trials are underway). A recent network metaanalysis ${ }^{53}$ found that "in the cluster analyses assessing safety and efficacy, apixaban, edoxaban and dabigatran ranked best followed by VKAs and rivaroxaban, whereas the WATCHMAN LAA closure device ranked last." Successful LAA occluder implantation converts the need for OAC to longterm antithrombotic treatment with aspirin and/or clopidogrel. For exactly this target group, there is a RCT that was prematurely stopped because of overwhelming superiority of apixaban in prevention of ischemic strokes with similar bleeding rates..$^{51}$ One could consider LAAO with all periprocedural risks, lack of non-inferiority compared to VKA, followed by VKA plus aspirin for 45 days or just dual antiplatelet regimen consisting of clopidogrel plus aspirin for 6 months followed by aspirin alone (if the procedure is successful, likely in $98 \%$ of cases with experienced interventionalist). One could consider directly taking a NOAC, which is non-inferior, and in some instances superior to VKA, which again (VKA) may theoretically represent a more favourable option than a LAA occluder, since the non-inferiority of WATCHMAN device compared to warfarin in long-term follow-up has not been established.

In any case, future trials should also systematically assess complications such as periprocedural strokes with magnetic resonance imaging ${ }_{1}^{54}$ efficacy issues in patients with persistent leakage, endocrinological aspects among others. Finally, patients not fitting the current approval such as patients with renal failure, previous intracranial bleedings, contraindications for OAC, and cerebral amyloid angiopathy should be recruited into future trials.

\section{Outlook and perspective}

On clinicaltrials.gov a search with condition as 'atrial fibrillation' and other keyword 'left atrial appendage' rendered 139 hits. Focussing on randomized trials, and excluding surgical or suture/clipping device trials, trials with additionally treated conditions such as coronary artery disease and registries or 
single arm studies, only a few relevant trials for the scope of this review remain. We found five trials in varying countries recruiting into trials comparing NOACs with LAA occluders, one of these for patients with hemorrhages, one for occluders versus VKA, one Chinese trial (WATCHMAN for Second Prevention of Stroke [WASPS]) comparing any antithrombotic regimen versus LAAO in 400 patients (not truly randomised). Two trials deserve a brief description.

(1) The Left Atrial Appendage CLOSURE in Patients With Atrial Fibrillation at High Risk of Stroke and Bleeding Compared to Medical Therapy: a Prospective Randomized Clinical Trial (CLOSURE AF trial) is an investigator initiated interventional randomised controlled trial aiming for 1,512 patients. The primary endpoint is net clinical benefit, which is a composite endpoint. It uses an event-driven group-sequential design, non-inferiority test, if this is significant it is followed by a superiority test. The trial compares CE marked LAA occluder devices versus standard (N)OAC/NKA treatments. The $\mathrm{CHA}_{2} \mathrm{DS}_{2}-$ VASc score has to be larger than 2. CLOSURE AF just started recruitment and plans completion in 2023.

(2) Another trial (industry sponsored) named "Assessment of the WATCHMAN ${ }^{\text {TM }}$ Device in Patients Unsuitable for Oral Anticoagulation (ASAP-TOO)" aims to establish the safety and effectiveness of the WATCHMAN including the post-implant medication regimen, for subjects with non-valvular AF who are deemed not to be eligible for anti-coagulation therapy to reduce the risk of stroke. It plans for recruitment of 888 patients until 2,023 randomized to WATCHMAN with modified postimplant regimen versus single antiplatelet or no therapy. Primary safety endpoint is a combined 7-day rate of death, ischemic stroke, systemic embolism, and complications requiring major cardiovascular or endovascular intervention. Primary efficacy endpoint is time to first event of ischemic stroke or systemic embolism with a maximum time frame of 5 years.

In conclusion, there appears to be merit and by now an established reasonable safety profile for device based (preferably WATCHMAN) occlusion of the LAA in patients with AF. While there is a somewhat restricted label and approval in some countries, several questions remain and ongoing trials have the chance to clarify these outstanding issues. The results of randomized trials, especially comparisons against NOACs-in keeping with the times-are dearly awaited. Until then we recommend either a restrictive indication of this procedure with FDA approved devices following the wording in the FDA approval, or recruitment into ongoing clinical trials. Off label use may be warranted in situations, where other therapeutic options are not feasible; however, this should follow an interdisciplinary decision process and very clear informative consent of the patients.

\section{Disclosure}

Peter D. Schellinger received honoraria, travel grants, and consulting fees from Boehringer Ingelheim, BMS-Pfizer, Daichi Sankyo, Bayer, and Medtronic. Georgios Tsivgoulis received honoraria and consulting fees from Boehringer Ingelheim, Daichi Sankyo, Bayer, Shire, and Medtronic. Thorsten Steiner received honoraria, travel grants, and consulting fees from Boehringer Ingelheim, BMS-Pfizer, Daichi Sankyo, Bayer, and Medtronic. Martin Köhrmann received honoraria, travel grants, and consulting fees from Boehringer Ingelheim, Daichi Sankyo, BMS-Pfizer, Bayer, and Medtronic.

\section{References}

1. Bonow RO, Carabello BA, Chatterjee $K$, de Leon AC Jr, Faxon $D P$, Freed MD, et al. 2008 Focused update incorporated into the ACC/AHA 2006 guidelines for the management of patients with valvular heart disease: a report of the American College of Cardiology/American Heart Association Task Force on Practice Guidelines (Writing Committee to Revise the 1998 Guidelines for the Management of Patients With Valvular Heart Disease): endorsed by the Society of Cardiovascular Anesthesiologists, Society for Cardiovascular Angiography and Interventions, and Society of Thoracic Surgeons. Circulation 2008;118:e523-e661.

2. Goette $A$, Bukowska $A$, Lendeckel U, Erxleben $M$, Hammwöhner $M$, Strugala $D$, et al. Angiotensin II receptor blockade reduces tachycardia-induced atrial adhesion molecule expression. Circulation 2008;117:732-742.

3. Di Biase L, Santangeli P, Anselmino M, Mohanty P, Salvetti I, Gili $S$, et al. Does the left atrial appendage morphology correlate with the risk of stroke in patients with atrial fibrillation? Results from a multicenter study. J Am Coll Cardiol 2012;60:531-538.

4. Al-Saady NM, Obel OA, Camm AJ. Left atrial appendage: structure, function, and role in thromboembolism. Heart 1999;82:547-554.

5. Blackshear JL, Odell JA. Appendage obliteration to reduce stroke in cardiac surgical patients with atrial fibrillation. Ann Thorac Surg 1996;61:755-759.

6. Mahajan R, Brooks AG, Sullivan T, Lim HS, Alasady M, Abed $H S$, et al. Importance of the underlying substrate in determining thrombus location in atrial fibrillation: implications for left atrial appendage closure. Heart 2012;98:1120-1126.

7. Kimura T, Takatsuki $S$, Inagawa $K$, Katsumata $Y$, Nishiyama $T$, Nishiyama N, et al. Anatomical characteristics of the left atrial appendage in cardiogenic stroke with low CHADS2 
scores. Heart Rhythm 2013;10:921-925.

8. Johnson WD, Ganjoo AK, Stone CD, Srivyas RC, Howard M. The left atrial appendage: our most lethal human attachment! Surgical implications. Eur J Cardiothorac Surg 2000;17:718722.

9. Patel MR, Mahaffey KW, Garg J, Pan G, Singer DE, Hacke W, et al. Rivaroxaban versus warfarin in nonvalvular atrial fibrillation. N Engl J Med 2011;365:883-891.

10. Connolly SJ, Ezekowitz MD, Yusuf S, Eikelboom J, Oldgren J, Parekh $A$, et al. Dabigatran versus warfarin in patients with atrial fibrillation. N Engl J Med 2009;361:1139-1151.

11. Granger CB, Alexander JH, McMurray JJ, Lopes RD, Hylek EM, Hanna $M$, et al. Apixaban versus warfarin in patients with atrial fibrillation. N Engl J Med 2011;365:981-992.

12. Boersma LV, Schmidt B, Betts TR, Sievert $H$, Tamburino $C$, Teiger $E_{1}$ et al. Implant success and safety of left atrial appendage closure with the WATCHMAN device: peri-procedural outcomes from the EWOLUTION registry. Eur Heart $J$ 2016;37:2465-2474.

13. Madden JL. Resection of the left auricular appendix; a prophylaxis for recurrent arterial emboli. J Am Med Assoc 1949; 140:769-772.

14. Contractor $T$, Khasnis A. Left atrial appendage closure in atrial fibrillation: a world without anticoagulation? Cardiol Res Pract 2011;2011:752808.

15. Healey JS, Crystal E, Lamy A, Teoh K, Semelhago L, Hohnloser $\mathrm{SH}$, et al. Left Atrial Appendage Occlusion Study (LAAOS): results of a randomized controlled pilot study of left atrial appendage occlusion during coronary bypass surgery in patients at risk for stroke. Am Heart J 2005;150:288-293.

16. Katz ES, Tsiamtsiouris T, Applebaum RM, Schwartzbard $A$, Tunick PA, Kronzon I. Surgical left atrial appendage ligation is frequently incomplete: a transesophageal echocardiograhic study. J Am Coll Cardiol 2000;36:468-471.

17. Kougioumtzoglou AM, Smith T, Swaans MJ, Boersma LVA, van Putte BP. Thoracoscopic left atrial appendage clipping as novel treatment option for peri-device leakage. Europace 2018;20:955.

18. Safavi-Naeini P, Rasekh A. Closure of left atrial appendage to prevent stroke: devices and status. Tex Heart Inst J 2018;45: 172-174.

19. Thakkar J, Vasdeki D, Tzikas A, Meier B, Saw J. Incidence, prevention, and management of periprocedural complications of left atrial appendage occlusion. Interv Cardiol Clin 2018;7:243-252.

20. Messas N, Ibrahim R. The amplatzer amulet device: technical considerations and procedural approach. Interv Cardiol Clin 2018;7:213-218.

21. Friedman PA, Holmes DR. Non-surgical left atrial appendage closure for stroke prevention in atrial fibrillation. J Cardiovasc Electrophysiol 2011;22:1184-1191.

22. Topcuoglu MA, Liu L, Kim DE, Gurol ME. Updates on prevention of cardioembolic strokes. J Stroke 2018;20:180-196.

23. Bayard $\mathrm{YL}$, Ostermayer $\mathrm{SH}$, Sievert $\mathrm{H}$. Transcatheter occlusion of the left atrial appendage for stroke prevention. Expert Rev Cardiovasc Ther 2005;3:1003-1008.

24. Ostermayer $\mathrm{SH}$, Reisman $\mathrm{M}$, Kramer PH, Matthews RV, Gray WA, Block PC, et al. Percutaneous left atrial appendage transcatheter occlusion (PLAATO system) to prevent stroke in high-risk patients with non-rheumatic atrial fibrillation: results from the international multi-center feasibility trials. J Am Coll Cardiol 2005;46:9-14.

25. Bayard $Y L$, Omran $H$, Neuzil $P$, Thuesen L, Pichler M, Rowland $E_{1}$ et al. PLAATO (Percutaneous Left Atrial Appendage Transcatheter Occlusion) for prevention of cardioembolic stroke in non-anticoagulation eligible atrial fibrillation patients: results from the European PLAATO study. Eurolntervention 2010;6:220-226.

26. Park JW, Bethencourt A, Sievert $H$, Santoro G, Meier B, Walsh $K_{1}$ et al. Left atrial appendage closure with Amplatzer cardiac plug in atrial fibrillation: initial European experience. Catheter Cardiovasc Interv2011;77:700-706.

27. Tzikas A, Shakir S, Gafoor S, Omran H, Berti S, Santoro G, et al. Left atrial appendage occlusion for stroke prevention in atrial fibrillation: multicentre experience with the AMPLATZER Cardiac Plug. Eurolntervention 2016;11:1170-1179.

28. Lam SC, Bertog S, Gafoor S, Vaskelyte L, Boehm P, Ho RW, et al. Left atrial appendage closure using the Amulet device: an initial experience with the second generation amplatzer cardiac plug. Catheter Cardiovasc Interv 2015;85:297-303.

29. Landmesser U, Tondo C, Camm J, Diener HC, Paul V, Schmidt $B$, et al. Left atrial appendage occlusion with the AMPLATZER Amulet device: one-year follow-up from the prospective global Amulet observational registry. Eurolntervention 2018; 14:e590-e597.

30. Nielsen-Kudsk JE, Johnsen SP, Wester P, Damgaard D, Airaksinen J, Lund J, et al. Left atrial appendage occlusion versus standard medical care in patients with atrial fibrillation and intracerebral haemorrhage: a propensity score-matched follow-up study. Eurolntervention 2017;13:371-378.

31. Cruz-Gonzalez I, Fuertes-Barahona M, Moreno-Samos JC, Gonzalez-Ferreiro R, Lam YY, Sanchez PL. Left atrial appendage occlusion: the current device landscape and future perspectives. Interv Cardiol Clin 2018;7:253-265.

32. Bergmann MW, Landmesser U. Left atrial appendage closure for stroke prevention in non-valvular atrial fibrillation: rationale, devices in clinical development and insights into im- 
plantation techniques. Eurolntervention 2014;10:497-504.

33. Bellmann B, Schnupp $S$, Kühnlein $P$, Javernik $C$, Kleinecke $C$, Rillig $A$, et al. Left atrial appendage closure with the New Occlutech $^{\circledast}$ device: first in man experience and neurological outcome. J Cardiovasc Electrophysiol 2017;28:315-320.

34. Park JW, Sievert H, Kleinecke C, Vaskelyte L, Schnupp S,

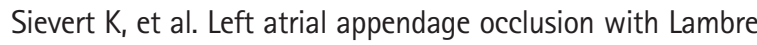
in atrial fibrillation: initial European experience. Int J Cardiol 2018;265:97-102.

35. Lakkireddy D, Afzal MR, Lee RJ, Nagaraj H, Tschopp D, Gidney $B$, et al. Short and long-term outcomes of percutaneous left atrial appendage suture ligation: results from a US multicenter evaluation. Heart Rhythm 2016;13:1030-1036.

36. Jazayeri MA, Vuddanda V, Turagam MK, Parikh V, Lavu M, Atkins $D$, et al. Safety profiles of percutaneous left atrial appendage closure devices: an analysis of the Food and Drug Administration Manufacturer and User Facility Device Experience (MAUDE) database from 2009 to 2016. J Cardiovasc Electrophysiol 2018;29:5-13.

37. Holmes DR, Reddy VY, Turi ZG, Doshi SK, Sievert H, Buchbinder $M$, et al. Percutaneous closure of the left atrial appendage versus warfarin therapy for prevention of stroke in patients with atrial fibrillation: a randomised non-inferiority trial. Lancet 2009;374:534-542.

38. Holmes DR Jr, Kar S, Price MJ, Whisenant B, Sievert H, Doshi $S K$, et al. Prospective randomized evaluation of the Watchman Left Atrial Appendage Closure device in patients with atrial fibrillation versus long-term warfarin therapy: the PREVAIL trial. J Am Coll Cardiol 2014;64:1-12.

39. Reddy VY, Holmes D, Doshi SK, Neuzil P, Kar S. Safety of percutaneous left atrial appendage closure: results from the Watchman Left Atrial Appendage System for Embolic Protection in Patients with AF (PROTECT AF) clinical trial and the continued access registry. Circulation 2011;123:417-424.

40. Boersma LV, Ince H, Kische S, Pokushalov E, Schmitz T, Schmidt $B$, et al. Efficacy and safety of left atrial appendage closure with WATCHMAN in patients with or without contraindication to oral anticoagulation: 1-year follow-up outcome data of the EWOLUTION trial. Heart Rhythm 2017;14:1302-1308.

41. Reddy VY, Sievert $H_{1}$ Halperin J, Doshi SK, Buchbinder M, Neuzil $P$, et al. Percutaneous left atrial appendage closure vs warfarin for atrial fibrillation: a randomized clinical trial. JAMA 2014;312:1988-1998.

42. Ezekowitz MD, Kent AP. Novel anticoagulants eliminate the need for left atrial appendage exclusion devices. Circulation 2014;130:1505-1514.

43. Maisel WH. Left atrial appendage occlusion: closure or just the beginning? N Engl J Med 2009;360:2601-2603.
44. McCabe DJ, Kinsella JA, Tobin WO. Left atrial appendage occlusion in non-valvular atrial fibrillation. Lancet 2009;374: 504-556.

45. Waksman R, Pendyala LK. Overview of the Food and Drug Administration circulatory system devices panel meetings on WATCHMAN left atrial appendage closure therapy. Am J Cardiol 2015;115:378-384.

46. Gangireddy SR, Halperin JL, Fuster V, Reddy VY. Percutaneous left atrial appendage closure for stroke prevention in patients with atrial fibrillation: an assessment of net clinical benefit. Eur Heart J 2012;33:2700-2708.

47. ACTIVE Investigators, Connolly SJ, Pogue J, Hart RG, Hohnloser $\mathrm{SH}$, Pfeffer $\mathrm{M}$, et al. Effect of clopidogrel added to aspirin in patients with atrial fibrillation. N Engl J Med 2009;360:20662078.

48. Reddy VY, Möbius-Winkler S, Miller MA, Neuzil P, Schuler G, Wiebe J, et al. Left atrial appendage closure with the Watchman device in patients with a contraindication for oral anticoagulation: the ASAP study (ASA Plavix Feasibility Study With Watchman Left Atrial Appendage Closure Technology). J Am Coll Cardiol 2013;61:2551-2556.

49. Yerasi C, Lazkani M, Kolluru P, Miryala V, Kim J, Moole H, et al. An updated systematic review and meta-analysis of early outcomes after left atrial appendage occlusion. J Interv Cardiol 2018;31:197-206.

50. Hanif H, Belley-Cote EP, Alotaibi A, Dvirnik N, Neupane B, Beyene J, et al. Left atrial appendage occlusion for stroke prevention in patients with atrial fibrillation: a systematic review and network meta-analysis of randomized controlled trials. J Cardiovasc Surg (Torino) 2018;59:128-139.

51. Connolly SJ, Eikelboom J, Joyner C, Diener HC, Hart R, Golitsyn $\mathrm{S}$, et al, Apixaban in patients with atrial fibrillation. $N$ Engl J Med 2011;364:806-817.

52. Reddy VY, Doshi SK, Sievert H, Buchbinder M, Neuzil P, Huber $\mathrm{K}$, et al. Percutaneous left atrial appendage closure for stroke prophylaxis in patients with atrial fibrillation: 2.3-year follow-up of the PROTECT AF (Watchman Left Atrial Appendage System for Embolic Protection in Patients with Atrial Fibrillation) trial. Circulation 2013;127:720-729.

53. Bajaj NS, Kalra R, Patel N, Hashim T, Godara H, Ather S, et al. Comparison of approaches for stroke prophylaxis in patients with non-valvular atrial fibrillation: network meta-analyses of randomized controlled trials. PLoS One 2016;11:e0163608.

54. Rillig A, Bellmann B, Skurk C, Leistner DM, Haeusler KG, Lin T, et al. Left atrial appendage angiography is associated with the incidence and number of magnetic resonance imagingdetected brain lesions after percutaneous catheter-based left atrial appendage closure. Heart Rhythm 2018;15:3-8. 